\title{
ACREDITACIÓN DE PROGRAMA DE POSTGRADO MIXTO (ACADÉMICO-PROFESIONAL) EN CHILE: IMPRESIONES ACADÉMICAS PARA UN DEBATE PENDIENTE
}

\author{
Christian Miranda J. (Uchile)*
}

\begin{abstract}
RESUMEN
El ensayo presenta impresiones académicas sobre los mecanismos de aseguramiento de la calidad de la Educación Superior, mediante el análisis histórico de la regulación universitaria y la conceptualización de los enfoques evaluativos vigentes en la materia. Además, relata las experiencias vividas por el autor, en su rol de Coordinador (20032011) del Programa mixto (académico-profesional) Magíster en Educación, mención Política y Gestión Educativas de la Universidad Austral de Chile ante la Comisión Nacional de Acreditación de Postgrado, deteniéndose en las implicancias prácticas y teóricas de la experiencia. Finalmente, concluye con algunas reflexiones exploratorias sobre la formación inicial y permanente de profesores y los desafíos pendientes en el debate académico nacional e internacional.

Palabras Claves: Educación superior. Acreditación de postgrado. Formación docente.
\end{abstract}

\begin{abstract}
ACCREDITATION OF MIXED (ACADEMIC AND PROFESSIONAL) GRADUATE PROGRAM IN CHILE: IMPRESSIONS FOR FAILED ACADEMIC DEBATE

The paper presents academic impressions on the mechanisms of guarantee of the quality of the Graduate Education, by means of the historical analysis of the university regulation and the conceptualization of the evaluative approaches in the matter. Besides, reports the experiences lived by the author, as Coordinator (2003-2011) of the Mixed Program (academic-professional) Master in Education, Political and Management mention of the Austral University of Chile by the National Commission of Accreditation of Post degree, in the practical and theoretical implications of the experience. Finally, concludes with some exploratory reflections on the teachers' initial and training formation and the hanging challenges in the academic national and international debate. Keywords: Superior education. Accreditation of postdegree. Teacher's formation.
\end{abstract}

\section{RESUMO}

ACREDITAÇÃO DO PROGRAMA DE PÓS-GRADUAÇÃO MISTO (ACADEMICOPROFISSIONAL) EM CHILE: IMPRESSÕES ACADEMICAS PARA UM DEBATE

$O$ ensaio apresenta vistas acadêmicas sobre os mecanismos de garantia da qualidade da Educação Superior de qualidade, através de uma análise histórica da regulação universidade e a conceituação dos enfoques avaliativos vigentes na matéria. Além

Doctor en Ciencias de la Educación en la Pontificia Universidad Católica de Chile. Coordinador del Magister en Educación, mención Informática Educativa. Hasta 2011 coordinator del Programa de Postgrado Mixto (Académico-Profesional) en Chile. Departamento de Educación - Facultad de Ciencias Sociales de la Universidad de Chile (FACSO/UChile). E-mail: christian.miranda@u.uchile.cl 
disso, relata as experiências do autor, em seu papel como Coordenador (2003-2011) do Programa misto (académica e profissional) Mestrado em Educação, Política Educativa e menção Política y Gestão educativa da Universidade Austral do Chile frente à Comissão Nacional de Acreditação graduado, refletindo nas implicações práticas e teóricas da experiência. Por fim, conclui com algumas reflexões exploratórias sobre a formação inicial e continuada dos professores e os desafios restantes no debate acadêmico nacional e internacional.

Palavras-chave: Ensino Superior. Acreditação de pos graduação. Formação de professores.

\section{Introducción}

La Universidad es una institución social y por lo tanto su organización y los principios de su funcionamiento están condicionados por las situaciones sociales en las qué surgió y se desarrolla. Se gestó como un medio para institucionalizar dos actividades humanas: la educación y la investigación, es decir, la preservación y la transmisión de la riqueza cultural y científica de la sociedad y la expansión de las exigencias científicas y teóricas por medio de la reflexión crítica y el enriquecimiento con nuevas ideas. ${ }^{1}$

Precisamente, nuevas ideas desde un certero diagnóstico es lo que proporcionaron los estudiantes secundarios chilenos hace diez años en la llamada Revolución Pingüina. Este movimiento permitió corroborar que se está en el umbral de una nueva etapa social. Se está en el preciso instante en que el país debe definir hacia donde quiere perfilar la calidad de vida de sus ciudadanos. La calidad de vida de un país se encuentra muy ligada a la calidad de su sistema educativo y, en éste último, tiene un papel prioritario la figura del profesor. Es este actor social quien, día a día, está en contacto con los estudiantes, el que asume la responsabilidad de formarlos y de asegurar el éxito de las escuelas.

En el contexto anterior, nuevamente 2 se hace el llamado a que son los maestros y maestras quienes deben poner al servicio de la enseñanza y el aprendizaje los muchos recursos que el proceso reformador ha puesto a disposición de los establecimientos educacionales (MIRANDA, 2007). Se

1 Artículo que forma parte del proyecto Fondecyt $\mathrm{N}^{\circ} 1140827$, financiado por CONICYT-Chile.

$2 \mathrm{La}$ adjetivación se explica dado que una constante de la reforma educativa actual ha sido el llamado a los docentes al protagonismo en la fase de instalación (1990-1995), desarrollo (1996-2000) y evaluación (2001-2006). insiste en que la calidad de la educación depende, en gran medida, de la calidad de los profesionales de la educación: los docentes. La renovada obligatoriedad de acreditación de las carreras de pedagogía, según se estipula en el proyecto de Ley de Educación Superior enviado al Congreso Nacional durante el presente año, y los argumentos de fortalecer el desarrollo profesional docente que aparecen en la Ley de Carrera Docente (MINISTERIO DE EDUCACIÓN DEL CHILE, 2016), no hacen más que avalar tal protagonismo profesional.

No obstante tales evocaciones y protagonismos, una cosa es incuestionable: el reconocimiento social de una profesión (si así se quiere llamar definitivamente al trabajo de los educadores), depende del nivel de cualificación exigido a quienes la practican. La fijación de Estándares para la Formación Inicial Docente (AVALOS, 2002), el Marco Para la Buena Enseñanza (MINISTERIO DE EDUCACIÓN DEL CHILE, 2003), los criterios para la Acreditación de las carreras de Pedagogía (COMISIÓN NACIONAL DE ACREDITACIÓN, 2010), las recomendaciones de la OCDE (2009) y propuestas actuales de desarrollo profesional docente del Centro de Perfeccionamiento, Experimentación e Investigaciones Pedagógicas (CENTRO DE PERFECCIONAMIENTO, EXPERIMENTACIÓN E INVESTIGACIÓN PEDAGÓGICA, 2015) ponen de manifiesto una preocupación compartida con la Comisión Nacional de Acreditación (COMISIÓN NACIONAL DE ACREDITACIÓN, 2015): la necesidad de contar con más reflexión teórica y antecedentes empíricos sobre el desarrollo de programas de postgrado en educación que articulen intereses 
académicos y profesionales mediante propuestas de formación docente de carácter mixto, dada su pertinencia en el mejoramiento requerido para el desarrollo profesional de los maestros y maestras en Chile. Este es el objetivo central del presente ensayo y, se espera, sea el horizonte que guía las reflexiones, avocadas a responder a las preguntas: ¿Cuáles son los antecedentes históricos, conceptuales y operacionales de los mecanismos de aseguramiento de la calidad que permiten mejorar la formación que reciben los docentes en su formación para responder a las nuevas demandas académicas y profesionales de una sociedad en cambio? y ¿Qué reflexiones deja la experiencia de coordinar un programa de naturaleza mixta como el Programa de Magíster en Educación, mención Política y Gestión Educativas de la Universidad Austral de Chile en términos teóricos y prácticos para el debate nacional e internacional de este tipo de propuestas formativas?

Para responder a tales preguntas el documento presenta, en un primer momento, los antecedentes históricos de la regulación universitaria, luego se precisa conceptual y operacionalmente la evaluación y los enfoques existentes sobre el tema de estudio. Además, se describen las vivencias del autor en la materia, a través de la experiencia de acreditar el Programa de Magíster antes mencionado, a fin de establecer las consecuencias teóricas y prácticas que ésta presenta en la formación de profesores y en el debate académico sobre la naturaleza mixta de tal propuesta formativa. Finalmente se reseña la bibliografía consultada.

\section{Antecedentes históricos de la regulación universitaria}

La calidad de la educación y los procesos de regulación en las instituciones de educación superior son elementos básicos desde su génesis. De acuerdo a la literatura consultada (BALART, 2003; LEMAITRE, 2005; PIZZA, 2000) se puede establecer a lo menos cuatro etapas. Estás, categorizadas históricamente coinciden con el Mundo Antiguo, Medieval, Moderno y Actual. En el Mundo Antiguo, el rol regulador lo ejercían los estudiantes o discípulos. Estos, al trasladarse, según referencias y cualidades de los maestros, establecían cuales eran los centros que daban garantías de calidad.

En las universidades medievales, la regulación se desplaza al gremio docente. En tal sentido la autorregulación según normas y procedimientos concordados por los profesores fue la forma de asegurarse cuotas de calidad.

En la época moderna, las instituciones de educación superior antiguas (en su mayoría) y nuevas entregaron el control de su organización al Estado. Esto por razones económicas y de cambio cultural. La excepción la constituyeron Instituciones americanas que mantuvieron la autonomía y libertad de enseñanza. No obstante lo anterior, el período se caracterizó porque el control, ya sea ministerial o establecido por la legislación nacional, fue ejercido por el Estado ${ }^{3}$ en materia curricular, financiera y de nombramiento académico. El ámbito regulatorio quedó relegado a un segundo plano, hasta avanzado el penúltimo decenio del siglo pasado.

En la actualidad, desde la década de los 80, como consecuencia de la expansión y diversificación de la educación superior, la internacionalización del espacio educativo y las demandas de una sociedad altamente globalizada, muchas universidades en los todos los continentes reestablecen en el debate académico los procedimientos de regulación, a fin de aumentar la eficiencia y la competencia, apelando para ello a la generación de una cultura evaluativa.

En síntesis, tanto en Chile como en muchos países del mundo se han iniciado procesos de regulación orientados a establecer mecanismos de aseguramiento de la calidad de la educación superior. Si bien la historia muestra que tales mecanismos forman parte del legado de cada etapa, en la actualidad los pasivos (deserción, desempleo y mercantilización) y activos (cobertura, diversificación y democratización) del sistema de educación superior chileno y demandas de la globalización e intereses nacionales o de bloques regionales como el Mercosur, los mecanismos de regulación se orientan a certificar calidad según parámetros internacionales. Aún más, tanto el Consejo Superior de Educación (CSE), mediante Seminarios Internacionales y publicación periódicas, como el Sistema

3 En Chile, la Universidad de Chile fue el organismo que ejerció mecanismos de regulación (grados y títulos) hasta la reforma de 1980 . 
de Aseguramiento de la Calidad de la Educación Superior (MINISTERIO DE EDUCACIÓN DEL CHILE, 2006b), buscan garantizar al público que se cumplan los objetivos enunciados, se entregue información suficiente y se satisfagan las necesidades y demandas de los usuarios en términos de perfil y desempeños deseados, mediante la instalación de una cultura de la evaluación.

\section{Concepto y enfoques de evaluación sobre la calidad de la educación superior}

La evaluación de la calidad de la educación superior presenta retos aún no resueltos: como por ejemplo, institucionalizar una cultura evaluativa y generar un modelo para evaluar su impacto. En tal sentido, como señala el Banco Mundial (2002, p. 19), "Los países en desarrollo y transición afrontan el riesgo de ser aún más marginados de la economía mundial, debido a que sus sistemas de educación superior no están adecuadamente preparados para capitalizar sobre la base de la creación y el uso del Conocimiento". Esto, según Pérez (2004), significa que no basta con políticas de aseguramiento de la calidad, si no se reflexiona sobre sus implicancias y sentidos.

¿Qué y cuándo se evalúa y quién debe hacerlo? Estas son preguntas propias de todos los procesos de evaluación, pero cuando se trata de la calidad de la educación superior, aparecen las primeras reacciones en contra de intervencionismo del Estado y mecanismos complejos que conllevan una sobrecarga para el personal responsable (KELLS, 2000). Es aquí donde los prejuicios se multiplican, se hace más difícil razonar sobre sus sentidos y el impacto parece diluirse. Es clave, entonces, formularse las interrogantes adecuadas, teniendo en cuenta los contextos de desarrollo de los mecanismos de evaluación diseñados.

Desde el punto de vista conceptual, la evaluación es un término susceptible de diferentes definiciones y a menudo intercambiable por otros en el ámbito educativo: medición, notas, pruebas, juicio de valor, rendición de cuentas, entre otras. La mayoría de las personas, asocia la evaluación con la valoración cuantitativa o cualitativa del rendimiento académico o profesional. No son pocos los que la siguen utilizando, sólo para categorizar a cada sujeto o programa en función de los resultados propios o en comparación a otros.

Tyler define la evaluación como el proceso surgido para determinar en qué medida han sido alcanzados los objetivos previamente establecidos. Años más tarde, el mismo autor empezó a prestar interés por la eficacia y el valor intrínseco de la evaluación para la mejora de la educación. Aparece un cierto desencanto con la educación pública y crece la presión por la rendición de cuentas (WHITFORD; JONES, 1998).

De esta forma, el concepto de evaluación amplía sus criterios valorativos. La evaluación educativa no sólo se relaciona con los resultados y mejora educativa, sino que se constituye en un proceso integrado de las acciones de enseñanza-aprendizaje, que debe satisfacer las necesidades y demandas de cada usuario.

Desde la esfera educativa se define a la evaluación como un proceso que lleva a emitir un juicio respecto de uno o más atributos de algo o alguien, fundamentado en información obtenida, procesada y analizada correctamente y contrastada con un referente claramente establecido, sustentado en un marco de referencia valórico y consistente con él, que está encaminado a mejorar los procesos educacionales y que produce efectos educativos en sus participantes, para lo que se apoya en el diálogo y la comprensión (HIMMEL, 2005).

Asumido el concepto y analizado desde el estado del arte sobre los mecanismos de aseguramiento de la calidad en la educación superior (COMISIÓN NACIONAL DE ACREDITACIÓN, 2011; CONSEJO SUPERIOR DE EDUCACIÓN, 2004; LEMAITRE, 2004; MARCHESI; MARTIN, 2000) y relativos a la formación de profesores en particular (COMISIÓN NACIONAL DE ACREDITACIÓN, 2015; CENTRO DE PERFECCIONAMIENTO, EXPERIMENTACIÓN E INVESTIGACIÓN PEDAGÓGICA, 2006; CONSEJO SUPERIOR DE EDUCACIÓN, 2001), la evaluación, más que un instrumento para controlar y reflexionar acerca de los procesos y resultados, se orienta a proporcionar evidencias sobre el ajuste a los propósitos declarados y a legitimar, frente a docentes y la sociedad en general, que lo aprendido es capaz por sí solo de justificar mecanismos de autorregulación 
en la materia y dejar atrás la forma tradicional de certificar calidad, mediante criterios de mercado o unidimensionales, confundiendo medios y fines y descuidando temas complejos como, por ejemplo, en el caso de la formación docente, la ausencia de conocimientos pedagógicos y experiencia empírica de aula de los formadores de profesores (ALVARADO, 2003).

Desde su irrupción en el mundo educativo y formativo, la evaluación ha generado importantes expectativas no sólo de carácter político, sino también de carácter social y económico, lo que, unido al creciente interés por la calidad educativa en cualquiera de sus manifestaciones y ámbitos, hace que se imponga la necesidad de desarrollar modelos de evaluación adecuados al objeto, sujeto y contexto en los que se producen (ARMANET, 2004).

En la literatura consultada se observa, según este autor, la presencia de dos enfoques de evaluación, orientados a la construcción de mecanismos de aseguramiento de la calidad de la educación superior en Chile, a saber:

a) El enfoque de diagnóstico-prescriptivo, que se orienta a evaluar los requisitos mínimos que debe poseer una institución para lograr su autonomía. $\mathrm{Su}$ objetivo es ejercer un control eminentemente externo, permanente y prolongado (seis años como mínimo) de los procesos involucrados en la gestión educativa, según el cumplimiento de sus objetivos, metas, rendimiento de los estudiantes, inserción profesional y la concreción de los perfiles de egreso entre otros; a fin de establecer estándares de calidad. En el país, se operacionaliza en a) la Verificación que aplica el Ministerio de Educación a los Centros de Formación Técnica (CFT), b) la Examinación, que se aplica a las instituciones creadas a partir de 1981, y c) el Licenciamiento 4 que aplica el CSE a los Institutos Profesionales y Universidades Privadas creados con posterioridad a la promulgación de la Ley Orgánica Constitucional de la Enseñanza (LOCE) en 1990.

b) El enfoque para la autorregulación y mejora, que busca estudiar los planes y desarrollos, donde los sistemas de control son esencialmente internos y participativos. Este tiene por objetivo evaluar el ajuste a los propósitos declarados, apoyándose en la evaluación externa y el seguimiento de la mejora.

4 Este se reconceptualiza a partir de la modificación del Artículo 55 de la Ley Orgánica Constitucional de Enseñanza de Chile, según Ley Nº 20.129.
Su propósito es consolidar los procedimientos que posibilitan la certificación de calidad formativa (expansión, diversificación y eficacia). En Chile, con la creación de la Comisión Nacional de Acreditación (CNA), se operacionaliza en tres mecanismos: a) Autoevaluación ${ }^{5}$ que aplica la propia institución siguiendo siete criterios establecidos por la CNA, b) Evaluación de Pares que emplean reconocidos académicos nacionales o internacionales que validan la información procedentes del Informe de Autoevaluación, y c) Acreditación que usa la CNA ${ }^{6}$ según los méritos de las instituciones o carreras, señalando además la vigencia de esta última en períodos de hasta siete años.

Discutiendo el aporte de tales perspectivas, se puede apreciar que la evaluación -ya sea diagnóstico-prescriptiva o de autorregulación-mejora ${ }^{7}$ - enfatiza el papel de la institución, de la evaluación como ajuste a los propósitos declarados y del Estado como evaluador calificado. Así, tal como lo sugieren los estudios en la literatura internacional (WOODHOUSE, 2004) y nacional (LEMAITRE, 2005) sobre los mecanismos de aseguramiento de la calidad, el centro está en la propia institución o programa y el ajuste a las acciones declaradas. Además, la evaluación no puede desarrollarse sin una relación con el contexto social en la que se pretende implementar. Finalmente, los principales enfoques de evaluación que subyacen al aseguramiento de la calidad de la educación superior, olvidan referirse a aspectos importantes. Aunque cada perspectiva evalúa dimensiones relevantes de las instituciones, lo hace de manera independiente. Y aunque se esfuerzan en optimizarlas, sólo la perspectiva de autorregulación-mejora permite abordar mejor

5 La Autoevaluación Institucional es un mecanismo central en los procesos de certificación de calidad actual. Prueba de ello es su alta tasa de aceptación a nivel nacional $(80 \%)$, a instancias de su voluntariedad inicial. Dado el énfasis del ensayo en la experiencia de acreditación y límites de este trabajo, el énfasis estará en la acreditación de postgrado.

6 En 1998, se crea la Comisión Nacional de Acreditación de Pregrado (CNAP) y de Postgrado (CONAP), ambas bajo la tutela del Ministerio de Educación chileno. En 2006 se crea la Comisión Nacional de Acreditación (CNA) que agrupa las comisiones anteriores en una sola orgánica. En la atualidad dado el cuestionamiento por conflicto de interés y crisis de confianza se ha enviado un proyecto de Ley para reemplazar la CNA por el Consejo para la Calidad de la Educación Superior. Ver Bachelet (2016).

7 Esta unión, para fines del presente estudio, se constituye en una enfoque holístico que recoge los lineamientos del enfoque de autorregulación y de mejora. 
las preguntas planteadas en este estudio, al ser un enfoque proactivo y eminentemente autogenerado, capaz de proporcionar las respuestas necesarias para considerar la autonomía de la Educación Superior y los actores que en ella participan. Como enfoque, proporciona un marco para reflexionar el modo en que el proceso de acreditación incide en el desarrollo de los Programas de Postgrado.

\section{Proceso de acreditación del Programa de Magister en Educación, Mención Política Y Gestión Educativas (MEPGE)}

La Universidad Austral de Chile (UACh) ${ }^{8}$ es una Institución tradicional, regional y compleja que ha definido como política institucional la acreditación de todos sus programas y carreras. Siguiendo parámetros internacionales y nacionales sobre regulación de la educación superior, satisfactorios procesos de acreditación institucional (2004-2009 y 2010-2016), de resultados observables derivados de proyectos de los Programas de Mejoramiento de la Calidad y Equidad de la Educación Superior (MECESUB) y enseñanzas de los procesos de acreditación de todos sus carreras y programas desde 1999, ha optado por institucionalizar la cultura evaluativa a través de la Oficina de Autoevaluación Institucional (OAI), dependiente de Vicerrectoría Académica.

En tal contexto, la UACh, a través de la OAI, hace suyos los objetivos específicos del Sistema de Aseguramiento de la Calidad de la Educación Superior, en términos de dar garantía pública de su calidad, promover y apoyar el mejoramiento de la educación superior, favorecer el establecimiento de una instancia de coordinación del sistema en su conjunto y procurar aportar en el desarrollo de programas y carreras que respondan a estándares nacionales e internacionales.

Bajo este marco, una vez acreditado el Postgrado (2004-2009) su Dirección, siguiendo las directrices de la Comisión Nacional de Acreditación de Postgrado e indicaciones de la OAI, comienza el proceso de acreditación de sus quince Programas, ${ }^{9}$

8 La Universidad Austral de Chile se crea el año 1954, en la cuidad de Valdivia (XV región), sur de Chile.

9 Si bien la acreditación de las carreras de Pregrado ha sido prioridad en la política de la UACh, este estudio se focaliza en el Postgrado, por ser la instancia en que la Institución, a juicio del autor, presenta resultados exitosos. Además, la incluyendo aquellos de corta historia.

Hasta el año 2015, la UACh ha acreditado sus seis Programas de Doctorado y trece de Maestría. El resto (cinco) se encuentra en proceso. En suma, la totalidad de los Programas de Doctorado y más de la mitad de los de Maestría están acreditados bajo las diferentes modalidades de tiempo que estipula la Comisión Nacional de Acreditación (que asume las funciones de la CONAP), acreditando programas de carácter académico y mixto. En este último caso, uno de los acreditados por mayor número de años es el Magister en Educación, mención Política y Gestión Escolar (En adelante MEPGE). ${ }^{10}$

El MEPGE se gesta bajo la lógica de restablecer la oferta académica para pedagogos ${ }^{11} \mathrm{y}$ la operacionalización del convenio establecido entre la Agencia Española de Cooperación Iberoamericana ${ }^{12}$ y la UACh, firmado en el segundo semestre del año 2003 (UNIVERSIDAD AUSTRAL DE CHILE, 2011). El diseño del Programa es aprobado para su ejecución inmediata por el Consejo de Facultad de Filosofía y Humanidades en el segundo semestre del 2004. Previa ratificación de la Dirección de Postgrado es decretada su existencia formal por el Consejo Superior de la UACh, el 03 de enero del 2005. ${ }^{13}$ Desde su primer año de ejecución, cuenta con el apoyo académico de la Universidad de Granada y Huelva (España), a los cuales se agregan docentes de Estados Unidos, Brasil y de universidades nacionales.

El Programa tiene por objetivo general formar profesionales que laboran en el sistema educativo con sólidos conocimientos científicos de índole teórico-práctico en gestión y política, posibilitándoles resolver problemas educativos atingentes a su labor al más alto nivel.

experiencia como Coordinador del MEPGE otorgaría mayor grado de validez a las reflexiones expresadas.

10 El MEPGE es acreditado por un periodo de 6 años (2011-2017), el máximo alcanzado por un programa de postgrado mixto en Educación a nivel nacional.

11 La UACh desde su origen, en 1956, forma profesores. Esto sumado a programas de Postgrado en educación desde 1979. Tal vocación pedagógica se vio abruptamente interrumpida en 1995, con la intervención de la Facultad de Filosofía y Humanidades y cierre de los programas de pedagogía, siguiendo criterios de mercado y de conflictos internos hasta hoy poco analizados.

12 Acogida en Convenio Universidad Austral - Consejo de Educación y Ciencias de la Junta de Andalucía (1996); Agencia Española de Cooperación Iberoamericana (AECI), resolución PCI IB03, Proyecto B/1118/03.

13 Decreto ${ }^{\circ}{ }^{3}$. Ver en Universidad Austral de Chile (2011). 
Si bien es cierto un Programa de Magíster en Educación no es algo nuevo ni exclusivo en el contexto nacional, su temática central - política y gestión educativas - le confiere un sello distintivo. A lo anterior se suma como aspecto relevante su carácter mixto (académico-profesional) y la realización de un Proyecto de Intervención Educativa, que sustituye la tradicional Tesis de Graduación.

El Programa es concebido como una opción que contribuye a mejorar los procesos de formación inicial docente en la continuidad de estudios de postgrado. ${ }^{14}$ Mediante esta modalidad de perfeccionamiento, la Universidad ofrece oportunidades de especialización a profesionales involucrados con los procesos de enseñanza/aprendizaje y a aquellos vinculados con la gestión y política, dando con ello respuesta a las demandas que surgen desde las instancias gubernamentales, académicas y sociales preocupadas por la educación (MINISTERIO DE EDUCACIÓN DEL CHILE, 2006a).

En el año 2011, luego de doce años de funcionamiento y un número significativo de graduados, ve sostenida su matrícula a 12 estudiantes en su treceava cohorte, lo cual muestra su grado de consolidación e impacto a nivel local y regional. Esta estabilidad significativa de la matrícula de estudiantes y la actual coyuntura educacional, ha propiciado adaptaciones del Programa en términos curriculares - enfatizando su carácter mixto y transdisciplinario - y mejoramiento de su planta docente. ${ }^{15}$ En el Grafico 1 se presenta el perfil profesional de los/ as estudiantes del MEPGE cohortes 2003-2011.

\section{Gráfico 1 - Perfil profesional del estudiantado del MEPGE}

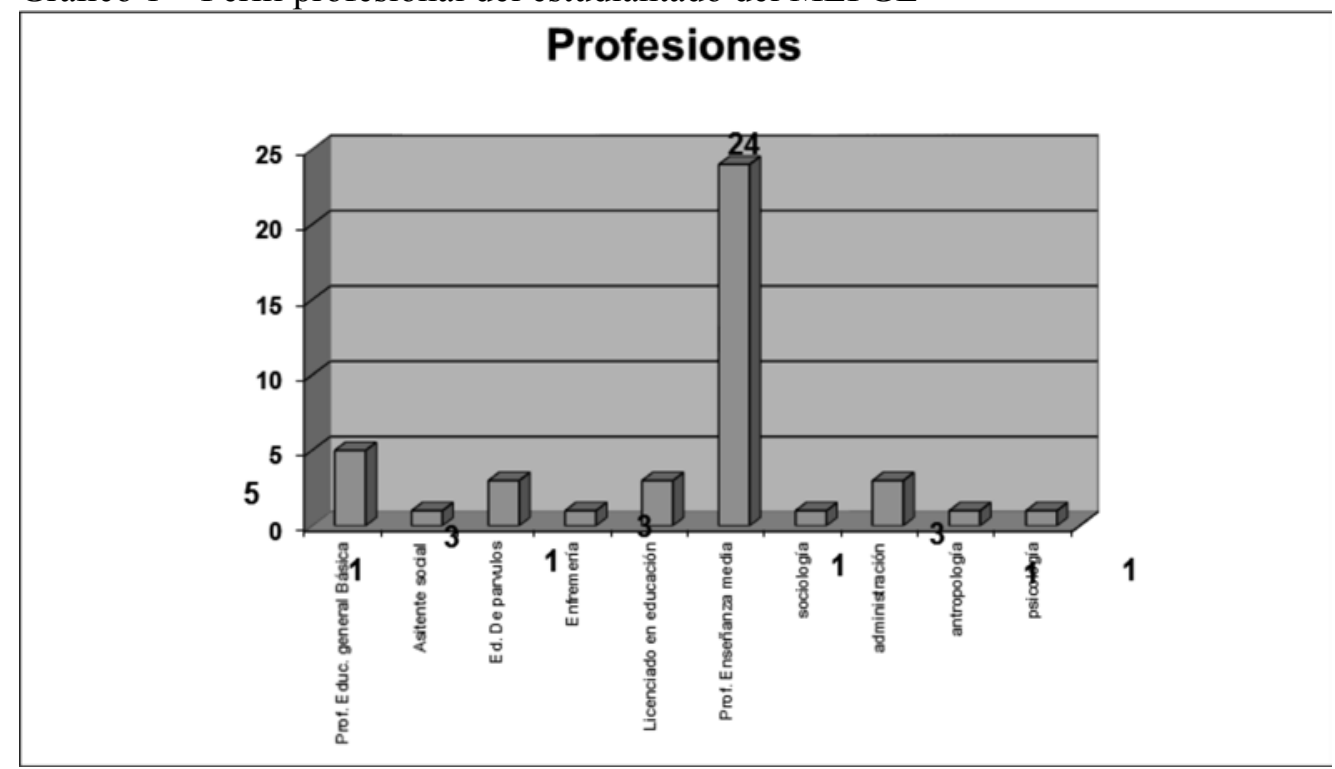

Fuente: Universidad Austral de Chile (2011).

Como se observa en el Grafico 1, las profesiones del estudiantado que ingresan al Programa provienen fundamentalmente del área Educacional $(81,4 \%)$. Dentro de ésta, el $57 \%$ tiene su origen en la pedagogía en educación media. La otra área relevante es administración, con un 12,6\%. Estás áreas se complementan con profesionales de la salud y ciencias sociales, con cifras que no son

14 El MEPGE considera el vínculo con el Pregrado mediante cursos estructurales (2) que remplazan a la Tesis, posibilitando el tránsito al Postgrado. estadísticamente significativas. Esto reafirma el énfasis de este artículo en su formación.

En este contexto, la Coordinación del Programa asume el desafió de re-acreditarlo. Lo hace bajo el supuesto que este proceso es un medio y no un fin en sí mismo. Es decir, sin perder de vista el desarrollo del Programa y sus actores involucrados. Además, el convencimiento que, lograda su con-

15 El Programa cuenta con 15 docentes (8 permanentes y 7 colaboradores); 13 de ellos tiene grado de Doctor y 2 el grado de Magíster y son candidatos a Doctor en universidades extranjeras. 
solidación regional, adecuada masa crítica y base institucional, se esta en condiciones de responder a la misión institucional (cultura evaluativa) y objetivos en orden a dar garantía pública de su calidad.

El proceso se inicia con una etapa de constitución del Comité de Autoevaluación, integrado por académicos y estudiantes. Luego de presenta ante la Escuela de Graduados e instancias institucionales el diseño del proceso, en sus tres fases: a) sensibilización y promoción ante la comunidad del Programa, b) recopilación de antecedentes cuali-cuantitativos, y c) análisis de datos y socialización de Informe. Sumado a lo señalado, se agregan reuniones con actores del sistema educativo, coordinadores de programas acreditados de la UACh y profesionales de la Oficina de Autoevaluación Institucional.

Una vez redactado el Borrador del Informe de Autoevaluación, se realiza una jornada ampliada con estudiantes y docentes del Programa, donde se socializan los activos y pasivos del proceso, concordando lo adecuado del mecanismo de aseguramiento utilizado en esta etapa y los desafíos que implica para el Coordinador en términos de gestión de tres instancias: Programa, Autoevaluación y Docencia, situación que requiere una revisión en términos humanos, académicos y evaluativos.

Bajo este diagnóstico compartido, se busca mejorar el Informe de Autoevaluación, siguiendo la lógica de los criterios establecidos por la CNA para programas mixtos ${ }^{16}$ que contempla como base conceptual el tratarse de programas que corresponden a estudios de nivel avanzado que procuran el desarrollo de competencias analíticas, sintéticas, de abstracción y de aplicación práctica. Se espera que el nivel de complejidad y profundización sea el sello que diferencia este nivel formativo del pregrado. Los programas junto con poder ser mixtos, pueden ser académicos y profesionales. Los criterios de evaluación son generales, esperándose en el caso de los programas mixtos un equilibrio entre lo académico y profesional, aspecto que si bien es referenciado en pautas de análisis es aun un aspecto

16 Estos son: propósitos institucionales, estructura organizacional y administrativa, perfil profesional del graduado y estructura curricular del programa, recursos humanos, efectividad del proceso de enseñanza-aprendizaje, infraestructura, apoyo técnico y recursos para la enseñanza e investigación y vinculación con el medio. crítico de conceptualizar y Operacionalizar en las propuestas que asumen este carácter.

Entre los criterios de evaluación de los programas mixtos se encuentran aquellos vinculados a dos bloques de variables, a saber: a) condiciones y dispositivos de formación (definición conceptual, contexto institucional, cuerpo académico, recursos de apoyo y capacidad de autorregulación) y b) Características y Resultados del Programa (carácter, objetivos, selección de postulantes, perfil de ingreso y egreso, estructura y plan de estudios y progresión de estudiantes)

Una vez analizada la información y contrastada con los criterios antes señalados, el paso siguiente es la elaboración del Plan de Desarrollo y Mejora, según los criterios precedentes y proponiendo líneas de acción, tiempo de concretización, indicadores de logro y responsable.

Tal como se estipula en la UACh, el Informe de Autoevaluación es visado por la Escuela de Graduados de la Facultad de Filosofía y Humanidades y revisado por la Oficina de Autoevaluación Institucional. Una vez validado en Informe, se envía a la Dirección de Postgrado, instancia que lo deriva al Comité Curricular para su evaluación interna definitiva. Una vez sancionado, se procede a contactar a destacados académicos en la temática para conformar el Comité de Pares académicos. Tal como se observa, las instancias de autorregulación son variadas y complejas, tanto desde el punto de vista temporal como burocrático, contemplando masas críticas especialistas en lo académico por sobre lo profesional o mixto, siendo este un punto crítico de este tipo de programas

El Comité de Pares académicos queda constituido finalmente por académicos nacionales que prestan servicios a las agencias acreditadoras, debidamente facultadas por la CNA. Estos realizan la visita que procede, recopilando información orientada a contrastar el ajuste a los propósitos declarados, según criterios establecidos por la CNA y percepción de actores involucrados, en orden a validar el Informe de Autoevaluación.

Es importante detenerse en este mecanismo de aseguramiento, dada su trascendencia en el caso del MEPGE y como ejemplo. Tanto la designación como la aprobación por parte de la CNA de los Pares son generalmente complejas, en especial 
cuando se trata de programas de carácter mixto. Sus razones principales: falta de pares profesionales acreditados en el área e interés de los mismos a participar en estos procesos que requiere tiempo, experticia y vocación de servicio. Hoy, a pesar que estos problemas están teórica y legalmente resueltos con las agencias acreditadoras y presentación de antecedentes para ser Evaluador Par incluidos en la Ley n ${ }^{\circ} 20.129$. Sin duda, el la falta de masa crítica que articule en su trayectoria antecedentes de calidad en lo académico y profesional, el carácter experimental de la inclusión de los programas mixtos y la inexperiencia confabulan también a tal problema.

Retomando el proceso, una vez desarrollada la visita, los Pares remiten el Informe de su evaluación del MEPGE, la cual es socializada a la comunidad académica y profesional que la conforma ${ }^{17}$. Este documento, tiene una relevancia sustancial en la "moral" de la comunidad de los programas, tales como el MEPGE al constatarse, según los Pares, sus principales debilidades estructurales, como por ejemplo para la frustrada acreditación del año 2005 , a saber: congruencia entre objetivos, perfil de egreso y malla curricular. Pese al golpe que significó tal incongruencia, el Comité de Autoevaluación y comunidad en general, en aquel entonces concordaron en que tal nudo crítico podría contra argumentarse argumentando respecto de la naturaleza y sentidos del carácter mixto del programa.

El Informe Final de Autoevaluación presenta una síntesis de los principales activos y pasivos del MEPGE, resaltando el carácter proactivo del mismo, las argumentaciones para certificar el ajuste académico-profesional a los propósitos declarados y matizaciones de las incongruencias develadas por los Pares. Una vez elaborado, se somete a los mecanismos de control interno descritos previamente, dictaminándose por parte de la Dirección de Postgrado su presentación inmediata ante la CNA. La razón esgrimida fue la consistencia encontrada en las argumentaciones sobre el carácter mixto del Programa. Si bien tiene sentido discutir las implicancias positivas de esta decisión en la

17 Resulta importante destacar este hecho. El carácter institucional de las visitas y su difusión (Pagina Web, Radio, publicaciones, entre otras) y la recurrente simultaneidad de procesos de acreditación le otorgan un carácter significativo a este proceso. comunidad del MEPGE, al no ser parte de este ensayo se omiten, quedando para futuros trabajos su abordaje. Sí es importante, para responder a la segunda pregunta del ensayo, reflexionar sobre las implicancias prácticas y teóricas de los mecanismos de aseguramiento de la calidad y las consecuencias para la formación docente.

\section{IMPLICANCIAS DEL PROCESO}

Las implicancias teóricas y prácticas del Proceso de Acreditación del MEPGE, según la reflexión del autor, están contenidas en los siguientes enunciados:

- Teóricas. Estas se pueden situar de acuerdo a Contexto. La necesidad de conocer los antecedentes históricos de la autorregulación, las funciones de la Educación Superior, la historia institucional y del Postgrado en Educación, a fin de tomar conciencia respecto de la importancia de la cultura evaluativa, el rol de las Universidades en la formación docente y la constitución de un modelo evaluativo transparente, fundamentado, con una base de datos homologables y que considere los recursos necesarios para el desarrollo y seguimiento del Proceso de Acreditación. Objeto. Situar a la evaluación como un componente esencial del proceso formativo y conocer los supuestos teóricos de la Reforma Educativa, sintetizados como ajuste a los propósitos declarados. Además, queda en evidencia la necesidad de discutir e investigar el impacto de los mecanismos de aseguramiento en el aprendizaje en la Educación Superior. - Sujeto. La centralidad de los profesores en el Proceso de Reforma y ser, a la vez, factor de causa y respuesta a los problemas del sistema educativo en general, no hacen más que avalar la necesidad de contar con más Programas de Maestría y Doctorados acreditados, situación que debe profundizarse teórica y legalmente desde la visión del docente como sujeto adulto y en proceso permanente de formación.

- Prácticas. Estas se pueden situar de acuerdo a La Educación Superior. La necesidad de 
contar con mecanismos de aseguramiento validados y empíricamente probados garantiza la legitimidad de los procesos, otorgando un sustento a la operatividad del Sistema de Aseguramiento en su conjunto. - La CNA. Como instancia estatal, debe ser considerada en la futura orgánica por su contribución en estos años de experimentación, tanto en sus fortalezas como debilidades. - La UACh. La operacionalización de la cultura evaluativa a través de la Oficina de Autoevaluación, sumado al Comité Curricular de Postgrado y otras instancias, si bien acrecienta los mecanismos de aseguramiento de la calidad, puede constituirse en una excesiva carga burocrática para el proceso de acreditación y ante la ausencia de masas críticas que contemplen académicos con destacada trayectoria profesional o de desarrollo mixto, ve limitada su validez para el análisis de programas como el MEPGE. En tal sentido, se observa positivamente la generación de una única orgánica, que considere la producción, socialización y difusión de información relativa a la calidad de las propuestas formativas, con presupuesto y personal con experiencia en el campo académico y profesional. - El MEPGE. La sistematización de la información, el conocimiento y apoyo de los actores involucrados se considera una oportunidad de hacer del Programa un instrumento de producción de conocimiento científico aplicado y evidencias empíricas para la toma de decisiones políticas y de gestión sobre la Educación, la formación docente y la propia Institución.

\section{Conclusiones}

Los antecedentes, argumentaciones y experiencias descritas en el artículo, evidencian la necesidad de mayor reflexión, investigación y evaluación de los mecanismos de aseguramiento de la calidad de la Educación Superior asociados a los programas de carácter mixto, dado su naturaleza aplicada y alcance experimental en el ámbito de la autorregulación y la acreditación a nivel nacional e internacional. La experiencia del autor a través de su rol como Coordinador, Presidente del Comité de Autoevaluación y docente del Programa de Magíster en Educación, mención Política y Gestión Educativas de la Universidad Austral de Chile, avalan tal aseveración.

El presente ensayo, al responder a una experiencia particular, no busca constituirse en un documento oficial, sino más bien contribuir al debate sobre el rol de la evaluación en la Educación Superior en general y de las tensiones entre lo académico y lo profesional en la formación docente en lo particular, con el propósito de exponer ante la comunidad académica brasileña los supuestos, principios y conocimientos que mueven al autor, sistematizados en la frase: "El país que queremos depende en gran medida del/la docente que formemos".

En tal sentido, las conclusiones esbozadas permitirán, probablemente, proporcionar reflexiones apropiadas para la toma de decisiones en a) la formación inicial y b) permanente de profesores.

a) Formación Inicial. El MEPGE, si bien es un Programa de Maestría pensado para docentes de aula con experiencia en gestión y política educativas, asume el desafío de ser parte del proceso terminal de las carreras de pedagogía de la UACh. El presente ensayo deja en evidencia la necesaria revisión del impacto de tal propuesta en el aprendizaje académico y profesional de los estudiantes y los necesarios ajustes al modelo. No obstante lo anterior, la introducción de esta posibilidad de culminación rápida y desafiante del Pregrado, constituye un aporte a su desarrollo. Además, el reconocimiento de la gestión y políticas como factores relevantes en la formación, ha nutrido de argumentos para el fortalecimiento del área pedagógica en los procesos curriculares de las pedagogías en la UACh. Por otro lado, el establecimiento de una cultura evaluativa a través del establecimiento de mecanismos de aseguramiento de la calidad internos y externos, ha impactado positivamente en la naturaleza mixta del programa, resituando el rol de la evaluación en los procesos formativos y de puente entre las clásicas dicotomías de la formación docente pedagogía-disciplina y teoría-práctica. Final- 
mente, a partir de la vinculación formación inicial y permanente del profesorado ha quedado en evidencia la existencia de modelos de formación distintos y de compleja complementariedad. Sin duda, esto último abre una línea de investigación aplicada orientada a develar los enfoques en uso en la formación docente y cómo ésta se profundiza en el aprendizaje profesional.

b) Formación Permanente. A partir del presente ensayo, se observa necesario centrar el debate en las concepciones, propuestas de acción y resultados de los Programas de Postgrado que, articulan aprendizajes complejos de carácter académico y profesional como el MEPGE, tienen por sujeto de formación a los/as docentes. Queda en evidencia el rol central que tiene ésta en la profesionalización temprana de los docentes noveles como de actualización de los experimentados. Además, en los campos académicos y profesionales, permite avanzar en el debate sobre la formación de los formadores, las experiencias exitosas de eficacia escolar y el necesario fortalecimiento del rol de la docencia en la Universidad, a lo menos en términos metodológicos, evaluativos y técnicos. Por otro lado, los académicos y profesionales que asumen los procesos de autoevaluación y acreditación, deben ser preparados y debidamente incentivados para el desafío que involucra tal tarea y carácter mixto. Finalmente, un elemento clave para el éxito del Programa es el vínculo con las comunidades acadé- micas y profesionales en las cuales labora el profesorado. Al respecto, se observa en el proceso de autoevaluación una compleja situación: los docentes cuentan con el tiempo para el aprendizaje profesional pero este al interferir en su quehacer, se asume como un tema personal y no institucional, es decir, pierde relevancia institucional y, por ende, se aminora el impacto que pudiera tener en términos de transferencia académica y de innovación profesional. Sin duda, se está haciendo camino, pero el impacto social que tiene la formación docente amerita no sólo la acreditación obligatoria de las carreras de pedagogía en Pregrado y de Postgrado, sino también una línea de investigación orientada a incluir en el debate nacional, de manera permanente, el tema de la naturaleza y sentido de los programas de postgrado de carácter mixto, dada su relevancia en el cambio educativo, constituyendo por ello un paso ineludible en materia de indagación y reflexión en contextos de reformas educativas de nivel terciario como el caso de Chile y Brasil.

Sin duda, el presente ensayo ha respondido exploratoriamente las preguntas planteadas. No obstante, deja en evidencia la importancia y trascendencia de la triada Educación Superior-mecanismos de aseguramiento de la calidad de programas mixtos-formación docente. En tal sentido, la acreditación en proceso del MEPGE es una muestra que la confluencia de ésta, es un debate pendiente a nivel internacional y nacional.

\section{REFERENCIAS}

ALVARADO, L. La Formación de Formadores. In: (Edit.). La formación permanente de profesores en diversos contextos latinoamericanos. Bogotá: Antropos, 2003. p. 7-55.

ARMANET, P. ¿Por qué Asegurar la Calidad? Revista Calidad de la Educación, Santiago, n. 21, p. 107-121, dec. 2004.

AVALOS, B. Profesores para Chile, historia de un proyecto. Santiago: MINEDUC, 2002.

BACHELET, M. Mensaje presidencial nº 110-364, del 4 de Julio de 2016. Proyecto de Ley de Educación Superior. Santiago, 2016.

BALART, C. Logro permanente de metas: un desafío para la formación de profesores. Contextos, Estudios de Humanidades y Ciencias Sociales, Santiago, n. 9, p. 13-20, 2003. 
BANCO MUNDIAL. Constructing knowledge societies: new challenges for tertiary education. Washington, D.C., 2002.

CENTRO DE PERFECCIONAMIENTO, EXPERIMENTACIÓN E INVESTIGACIÓN PEDAGÓGICA(CPEIP). Formación Continua de Docentes: un camino para compartir (2000-2005). Santiago: MINEDUC, 2006.

Desarrollo profesional docente en Chile. Documento de trabajo. Santiago, 2015.

COMISIÓN NACIONAL DE ACREDITACIÓN (CNA). Criterios de evaluación de carreras profesionales, carreras profesionales con licenciatura y programas de licenciatura. Santiago, 2010.

Cuenta Pública 2010. Santiago, 2011.

Cuenta Pública 2015. Santiago, 2015.

CONSEJO SUPERIOR DE EDUCACIÓN (CSE). Educación Superior: perfil profesional del profesor. Santiago, 2001.

El Aseguramiento de la Calidad de la Educación Superior. Santiago, 2004.

HIMMEL, E. Discurso inaugural. In: CONSEJO NACIONAL DE EDUCACIÓN (CNE). Aseguramiento de la calidad: impacto y proyecciones. Santiago: CSE, 2005. p. 17-18.

KELLS, H. Autorregulación en la Educación Superior Chilena. Santiago: CSE, 2000.

LEMAITRE, M. Mecanismos de aseguramiento de la calidad: respuesta a los desafíos del cambio en la educación superior. Revista Calidad de la Educación, Santiago, n. 21, p. 87-106, dec. 2004.

. Aseguramiento de la calidad en Chile: impacto y proyecciones. In: CONSEJO NACIONAL DE EDUCA$\overline{\mathrm{CIÓN}}$ (CNE). Aseguramiento de la Calidad: impacto y proyecciones. Santiago: CNE, 2005. p. 55-69.

MARCHESI, A.; MARTÍN, E. Calidad de la enseñanza en tiempos de cambio. Madrid: Alianza Editorial, 2000.

MINISTERIO DE EDUCACIÓN DEL CHILE (MINEDUC). Marco para la Buena Enseñanza. Santiago, 2003.

Informe Final del Consejo Asesor Presidencial para la Calidad Educativa. Santiago, 2006a.

. Ley n ${ }^{\circ} 20.129$. Del Sistema de Aseguramiento de la Calidad de la Educación Superior. Santiago, $2006 \mathrm{~b}$.

Ley $n^{\circ}$ 20.203. Sistema de Desarrollo Profesional Docente. Santiago, 2016.

MIRANDA, C. H. Educación superior, mecanismos de aseguramiento de la calidad y formación docente en Chile: un debate pendiente. Estudios Pedagógicos, v. 33, n. 1, p. 95-108, 2007.

ORGANIZACIÓN PARA LA COOPERACIÓN Y EL DESARROLLO ECONÓMICOS (OCDE). Informe del Sistema Educativo Chileno. Santiago: MINEDUC, 2009.

PÉREZ, M. Aseguramiento de la Calidad de la Educación Superior en América Latina: ¿vamos por el camino correcto? Revista Calidad de la Educación, n. 21, p. 273-285, 2004.

PIZZA, M. Calidad en la Educación Superior: procesos de supervisión y acreditación. In: CONSEJO SUPERIOR DE EDUCACIÓN (CSE.) Políticas de Educación Superior: ¿Tiempo de innovar? Santiago, 2000. p. 120-123.

UNIVERSIDAD AUSTRAL DE CHILE (UACh). Informe de autoevaluación del Programa de Magister en Educación, mención Política y Gestión Educativas. Anexo 1. Valdivia, 2011.

WITHFORD, B.; JONES, K. Assessment and Accountability in Kentucky: How High Stakes Affects Teaching and Learning. In: HARGREAVES, A. (Ed.). International Handbook of Educational Change. Gran Bretaña: Kluwer Academic Publishers, 1998. p. 1163-1178.

WOODHOUSE, D. Desarrollo Global del Aseguramiento de la Calidad. Revista Calidad de la Educación, Santiago, n. 21, p. 17-36, dec. 2004. 